\title{
Increased vancomycin minimum inhibitory concentration among Staphylococcus aureus isolates in Malaysia
}

Vancomycin is one of the very few antibiotics available for the treatment of meticillin-resistant Staphylococcus aureus (MRSA). A shift towards higher

vancomycin MIC values has been observed among meticillin-susceptible and -resistant S. aureus strains (Wang et al., 2006;

Steinkraus et al., 2007). This increase in

MICs over time, referred to as 'MIC creep', is usually not recognized because these small increases are still within susceptible limits unless analyses are carried out to see the increasing trend over the years.

However, even small increases in MIC below the susceptibility breakpoint can affect the clinical efficacy (Sakoulas et al., 2004; Moise \& Schentag, 2000).

In Malaysia, $26 \%$ of S. aureus isolates from clinical specimens in 2008 were MRSA. Out of 2389 cases of bacteraemia due to $S$. aureus in 2008, $21 \%$ were caused by MRSA. S. aureus is also the second most common bacterium isolated from blood (Ministry of Health, 2008). Heterogeneous vancomycin-sensitive S. aureus, vancomycin-intermediate $S$. aureus and vancomycin-resistant $S$. aureus have not been documented in Malaysia; however, vancomycin treatment failure in vancomycin-susceptible MRSA has been reported and its occurrence may not be uncommon (Norazah et al., 2009). Vancomycin MIC determination is not routinely carried out on clinical isolates of MRSA unless requested by the clinicians when their patients did not respond to treatment. Thus the distribution of vancomycin MIC values among the $S$. aureus isolates in our hospitals is unknown. We conducted this study to determine the distribution of vancomycin MIC values among meticillin-susceptible S. aureus (MSSA) and MRSA isolates from six major Malaysian hospitals using a broth microdilution (BMD) method and Etest.

S. aureus strains were collected from six major government hospitals, namely Kuala
Lumpur Hospital (KLH), Selayang Hospital (SH), Penang Hospital (PH), Raja Perempuan Zainab II Hospital (RPZH), Sultanah Aminah Hospital (SAH) and Queen Elizabeth Hospital (QEH). All hospitals are located in West Malaysia, except QEH, which is located in East Malaysia, separated by the Straits of Malacca. These hospitals have 700-1000 beds. Only strains that were suspected pathogens (no isolates from nasopharyngeal carriage surveys, normal flora or environmental isolates) and only one isolate per patient were included in this study. The strain collection was terminated when a total of 300 MSSA and 300 MRSA strains were obtained, which took a duration of 5 months between March and July 2009. At the Bacteriology Unit, Institute for Medical Research, the $S$. aureus strains were reconfirmed using standard methods and meticillin resistance was determined using $30 \mu \mathrm{g}$ cefoxitin discs as outlined by the Clinical and Laboratory Standards Institute (CLSI, 2009). Isolates were stored frozen at $-70{ }^{\circ} \mathrm{C}$ in tryptic soy broth with $15 \%$ glycerol and subcultured twice to determine purity, prior to being tested.

The vancomycin MIC testing was carried out using the BMD method and Etest. The BMD method was carried out following CLSI guidelines (CLSI, 2006). The vancomycin concentration tested ranged from 0.25 to $8 \mu \mathrm{g} \mathrm{ml}^{-1}$. Etest was carried out using vancomycin Etest strips (MIC range $0.016-256 \mu \mathrm{g} \mathrm{ml}^{-1}$ ), following the manufacturer's instructions $(\mathrm{AB}$ BIODISK). Both methods were carried out by a single operator. MSSA ATCC 29213 and MRSA ATCC 33591 were used as controls.

Pearson's chi-square test was used to determine the significance between the vancomycin MIC values of MSSA and MRSA using either the BMD or Etest methods. The same test was also used to determine the significance of vancomycin
MIC in bacteraemia and non-bacteraemia isolates. SPSS version 15.0 was used for the analysis.

Seventy-one strains (23 MSSA; 48 MRSA) were isolated from KLH; 164 (43 MSSA; 121 MRSA) from SH; 29 (17 MSSA; 12 MRSA) from PH; 120 (98 MSSA; 22 MRSA) from RPZH; 152 (82 MSSA; 70 MRSA) from SAH; and 64 (37 MSSA; 27 MRSA) from QEH.

Forty per cent of the S. aureus strains (106 MSSA and 131 MRSA) were isolated from blood, $12 \%$ were from wound swabs, $6 \%$ from respiratory specimens, $2.5 \%$ from urine, $25.5 \%$ from superficial and deep skin infection, $10.5 \%$ from tissue, $2 \%$ from cerebrospinal fluid and peritoneal fluids and $1.5 \%$ from other samples.

By the BMD method, a significant difference was observed where MRSA strains had a higher MIC value of $1 \mu \mathrm{g}$ $\mathrm{ml}^{-1}$ compared to MSSA strains $(P<0.05)$ while the lower MIC of $<1 \mu \mathrm{g} \mathrm{ml}^{-1}$ was observed mainly among MSSA strains $(P<0.05)$ (Table 1).

By Etest, the majority of the MSSA and MRSA strains had a vancomycin MIC of $1.5 \mu \mathrm{g} \mathrm{ml}^{-1}$ and twice the number of MRSA strains than MSSA strains showed a vancomycin MIC of $2 \mu \mathrm{g} \mathrm{ml}^{-1}$ (30\% and $16 \%$, respectively). MRSA strains were also shown to have significantly higher vancomycin MIC values of $>1 \mu \mathrm{g} \mathrm{ml}^{-1}$ than MSSA strains when Etest was used $(P<0.05)$. Among the strains isolated from blood, 39\% (51/131) of MRSA strains had a vancomycin MIC of $2 \mu \mathrm{g} \mathrm{ml}^{-1}$ compared to only $13 \%(14 / 106)$ of MSSA strains $(P<0.05)$. Among non-blood MRSA isolates, $24 \%(40 / 169)$ had a vancomycin MIC of $2 \mu \mathrm{g} \mathrm{ml}^{-1}$ compared to $18 \%$ (34/ $194)$ among non-blood MSSA isolates $(P<0.05)$.

In this study, the determination of vancomycin MIC by the BMD method and Etest gave different values for MSSA and MRSA strains. Both the MSSA and MRSA 
Table 1. Vancomycin MIC of $S$. aureus isolates by the broth microdilution (BMD) method and Etest

\begin{tabular}{|c|c|c|c|c|}
\hline \multirow{2}{*}{ Vancomycin MIC $\left(\mu \mathrm{g} \mathrm{ml}^{-1}\right)$} & \multicolumn{2}{|c|}{$\operatorname{MSSA}[n(\%)]$} & \multicolumn{2}{|c|}{ MRSA [n (\%)] } \\
\hline & BMD & Etest & BMD & Etest \\
\hline 0.25 & $1(0.3)$ & 0 & $7(2.3)$ & $0(0)$ \\
\hline 0.5 & $261(87)$ & 0 & $171(57)$ & $6(2)$ \\
\hline 0.75 & & $2(0.6)$ & & $9(3)$ \\
\hline 1 & $38(12.7)$ & $101(33.7)$ & $122(40.7)$ & $40(13.3)$ \\
\hline 1.5 & & $149(49.7)$ & & $154(51.3)$ \\
\hline 2 & 0 & $48(16)$ & 0 & $91(30.3)$ \\
\hline
\end{tabular}

strains gave twofold or higher MIC values by Etest than by the BMD method. This is in concordance with other studies which showed that higher MIC values were obtained when Etest was used (Sader et al., 2009; Prakash et al., 2008; Hsu et al., 2008). A higher percentage of MRSA strains than MSSA strains were shown to have a vancomycin MIC of $2 \mu \mathrm{g} \mathrm{ml}^{-1}$. This was also observed among bacteraemia isolates of MRSA.

In the BMD method, twofold dilutions were used in the MIC testing and therefore strains with intermediate MIC could not be detected. In contrast, Etest, which incorporates intermediate as well as the traditional dilutions, allows the detection of strains with intermediate MIC values. In this study, the majority of strains showed MIC values of $1.5 \mu \mathrm{g} \mathrm{ml}^{-1}$ by Etest. This method has also been shown to be more sensitive and specific in identifying MRSA strains with reduced susceptibility to vancomycin when compared with the standard BMD test and automated tests (VITEK and MicroScan) (Walsh et al., 2001). Hsu et al. (2008) showed that Etest MIC results appear to be more precise and predictive of treatment response than results obtained by the standard twofold dilution method.

Increasing vancomycin MIC within the susceptible range was associated with a significant risk for vancomycin treatment failure in MRSA bacteraemia (Sakoulas et al., 2004). Higher clinical failure in MRSA bacteraemia was noted with vancomycin MIC $\geqslant 1.5 \mu \mathrm{g} \mathrm{ml}^{-1}$ (Lodise et al., 2008; Soriano et al., 2008). A higher failure rate was associated with strains with vancomycin MIC of $\geqslant 1.5 \mu \mathrm{g} \mathrm{ml}^{-1}$ compared to those with MICs $\leqslant 1 \mu \mathrm{g} \mathrm{ml}^{-1}$ by Etest (Lodise et al., 2008). MRSA isolates that show reduced susceptibility to vancomycin (with an MIC at the breakpoint for susceptibility) are highly prevalent among strains that cause invasive infections (Hidayat et al., 2006). In our study, a greater proportion of isolates with vancomycin MIC $2 \mu \mathrm{g} \mathrm{ml}^{-1}$ than of lowMIC strains were involved in bloodstream infections. Even though in this study we did not correlate the clinical outcome of MRSA bacteraemia, the possibility of vancomycin treatment failures in our hospital settings could not be ruled out and is an area of concern. The presence of subpopulations of resistant strains is also a possible cause for vancomycin treatment failure for the seemingly susceptible MRSA strains. These strains are difficult to detect and appear fully susceptible when tested by the BMD method or Etest. Vancomycin has been the main antibiotic used for the treatment of serious MRSA infections, while $\beta$-lactam antibiotics still remain the treatment of choice for MSSA infections. Prior recent exposure to vancomycin has been associated with reduced susceptibility of MRSA bloodstream isolates and increased tolerance to vancomycin in vitro (Moise et al., 2008). In vitro evaluations have demonstrated a relationship between exposure to low vancomycin serum concentrations and the development of heteroresistant vancomycin-intermediate significant percentage of MRSA bloodstream isolates with a high MIC of $2 \mu \mathrm{g} \mathrm{ml}^{-1}$ in this study would be of clinical relevance if prior exposure to vancomycin is known. Knowledge of previous vancomycin therapy in patients with MRSA bacteraemia could guide the physicians into considering alternative treatment when dealing with vancomycin treatment failure cases. S. aureus (Liu \& Chambers, 2003). The
Increasing MIC values or vancomycin MIC creep have been observed among $S$. aureus strains over the years in several institutions (Wang et al., 2006; Steinkraus et al., 2007; Robert et al., 2006; Ho et al., 2010).

Isolates with vancomycin MICs of $\geqslant 1 \mu \mathrm{g}$ $\mathrm{ml}^{-1}$ tend to be less susceptible to other antimicrobial agents (Wang et al., 2006). In this study, we noted a high percentage of our MRSA strains with MICs $1 \mu \mathrm{g} \mathrm{ml}^{-1}$ by the BMD method and $1.5 \mu \mathrm{g} \mathrm{ml}^{-1}$ by Etest. As the efficacy of vancomycin in treating $S$. aureus infections decreased with vancomycin MICs of $\geqslant 1 \mu \mathrm{g} \mathrm{ml}^{-1}$, the observed MIC among our local strains is indeed worrying. Even though the MIC values were dependent on the methods used, the high MICs of $\geqslant 1 \mu \mathrm{g} \mathrm{ml}^{-1}$ observed among our strains is a cause for concern as this may have an impact on success of treatment with vancomycin.

\section{Acknowledgements}

We would like to thank the Director General of Ministry of Health Malaysia for permission to publish this article.

\section{Norazah Ahmad, ${ }^{1}$ Salbiah Nawi, ${ }^{2}$ Ganeswrie Rajasekaran, ${ }^{3}$ Nurahan Maning, 4 \\ Mohamad Nazri Aziz, ${ }^{5}$ Azura Husin ${ }^{6}$ and Nor Iza Abdul Rahman ${ }^{7}$}

\section{${ }^{1}$ Bacteriology Unit, Institute for Medical Research, Kuala Lumpur, Malaysia \\ ${ }^{2}$ Selayang Hospital, Selangor, Malaysia \\ ${ }^{3}$ Sultanah Aminah Hospital, Johor, Malaysia \\ ${ }^{4}$ Raja Perempuan Zainab II Hospital, Kelantan, Malaysia \\ ${ }^{5}$ Kuala Lumpur Hospital, Kuala Lumpur, Malaysia \\ ${ }^{6}$ Queen Elizabeth Hospital, Sabah, Malaysia \\ ${ }^{7}$ Penang Hospital, Penang, Malaysia \\ Correspondence: Norazah Ahmad (norazah@imr.gov.my)}

CLSI (2006). Methods for Dilution Antimicrobial Susceptibility Tests for Bacteria that Grow Aerobically; Approved Standard, 7th edn, M7A7, vol. 26 no. 2. Wayne, PA: Clinical and Laboratory Standards Institute.

CLSI (2009). Performance Standards for Antimicrobial Susceptibility Testing, 19th Informational Supplement. M100-S19, vol. 29 
no. 3. Wayne, PA: Clinical and Laboratory Standards Institute.

Hidayat, L. K., Hsu, D. I., Quist, R., Shriner, K. A. \& Wong-Beringer, A. (2006). High-dose vancomycin therapy for methicillin-resistant Staphylococcus aureus infections: efficacy and toxicity. Arch Intern Med 166, 2138-2144.

Ho, P. L., Lo, P. Y., Chow, K. H., Lau, E. H., Lai, E. L., Cheng, V. C. \& Kao, R. Y. (2010).

Vancomycin MIC creep in MRSA isolates from 1997 to 2008 in a healthcare region in Hong Kong. J Infect 60, 140-145.

Hsu, D. I., Hidayat, L. K., Quist, R., Hindler, J., Karslsson, A., Yusof, A. \& Wong-Beringer, A.

(2008). Comparison of method-specific vancomycin minimum inhibitory concentration values and their predictability for treatment outcome of methicillin-resistant Staphylococcus aureus (MRSA) infections. Int J Antimicrob Agents 32, 378-385.

Liu, C. \& Chambers, H. F. (2003). Staphylococcus aureus with heterogeneous resistance to vancomycin: epidemiology, clinical significance, and critical assessment of diagnostic methods. Antimicrob Agents Chemother 47, 3040-3045.

Lodise, T. P., Graves, J., Evans, A., Graffunder, E., Helmecke, M., Lomaestro, B. M. \&

Stellrecht, K. (2008). Relationship between vancomycin MIC and failure among patients with methicillin-resistant Staphylococcus aureus bacteraemia treated with vancomycin. Antimicrob Agents Chemother 52, 33153320.
Ministry of Health (2008). National Surveillance of Antibiotic Resistance Report. Malaysia: Ministry of Health. http://www.imr.gov.my/ report/nsar.htm

Moise, P. A. \& Schentag, J. J. (2000).

Vancomycin treatment failures in Staphylococcus aureus lower respiratory tract infections. Int $J$ Antimicrob Agents 16, S31-S34.

Moise, P. A., Smyth, D. A., El-Fawal, N., Robinson, D. A., Holden, P. N., Forrest, A. \& Sakoulas, G. (2008). Microbiological effects of prior vancomycin use in patients with methicillin-resistant Staphylococcus aureus bacteraemia. J Antimicrob Chemother 61, 85-90.

Norazah, A., Salbiah, N., Nurizzat, M. \&

Santhana, R. (2009). Vancomycin treatment failure in a vancomycin susceptible methicillinresistant Staphylococcus aureus (MRSA) infected patient. Med J Malaysia 64, 166-167.

Prakash, V., Lewis, J. S. \& Jorgensen, J. H. (2008). Vancomycin MICs for methicillinresistant Staphylococcus aureus isolates differ based upon the susceptibility test method used. Antimicrob Agents Chemother 52, 4528.

Robert, J., Bismuth, R. \& Jarlier, V. (2006). Decreased susceptibility to glycopeptides in methicillin-resistant Staphylococcus aureus: a 20 year study in a large French teaching hospital, 1983-2002. J Antimicrob Chemother 57, 506-510.

Sader, H. S., Rhomberg, P. R. \& Jones, R. N. (2009). Nine-hospital study comparing broth microdilution and Etest method results for vancomycin and daptomycin against methicillin-resistant Staphylococcus aureus. Antimicrob Agents Chemother 53, 3162-3165.

Sakoulas, G., Moise-Broder, P. A., Schentag, J., Forest, A., Moellering, R. C. \& Eliopoulos, G. M. (2004). Relationship of MIC and bactericidal activity to efficacy of vancomycin for treatment of methicillin-resistant Staphylococcus aureus bacteraemia. J Clin Microbiol 42, 2398-2402.

Soriano, A., Marco, F., Martinez, J. A., Pisos, E., Almela, M., Dimova, V. P., Alamo, D., Ortega, M., Lopez, J. \& Mensa, J. (2008). Influence of vancomycin minimum inhibitory concentration on the treatment of methicillin-resistant Staphylococcus aureus bacteraemia. Clin Infect Dis 46, 193-200.

Steinkraus, G., White, R. \& Friedrich, L. (2007). Vancomycin MIC creep in non-vancomycinintermediate Staphylococcus aureus (VISA), vancomycin-susceptible clinical methicillinresistant $S$. aureus (MRSA) blood isolates from 2001-05. J Antimicrob Chemother 60, 788-794.

Walsh, T. R., Bolmström, A., Qwärnström, A., Ho, P., Wootton, M., Howe, R. A., MacGowan, A. P. \& Diekema, D. (2001). Evaluation of current methods for detection of staphylococci with reduced susceptibility to glycopeptides. $J$ Clin Microbiol 39, 2439-2444.

Wang, G., Hindler, J. F., Ward, K. W. \& Bruckner, D. A. (2006). Increased vancomycin MICs for Staphylococcus aureus clinical isolates from a university hospital during a 5-year period. J Clin Microbiol 44, 3883-3886. 